\title{
Weak Insertion of a Perfectly Continuous Function
}

\author{
MAJID MIRMIRAN* \\ Department of Mathematics, University of Isfahan, Iran.
}

*Corresponding Author: MAJID MIRMIRAN, Department of Mathematics, University of Isfahan, Iran

\begin{abstract}
A sufficient condition in terms of lower cut sets are given for the insertion of a perfectly continuous function between two comparable real-valued functions on such topological spaces that 1 -sets are open.
\end{abstract}

\section{INTRODUCTION}

A generalized class of closed sets was considered by Maki in 1986 [10]. He investigated the sets that can be represented as union of closed sets and called them $V$-sets. Complements of $V$-sets, i. e., sets that are intersection of open sets are called $\Lambda$-sets [10].

Recall that a real-valued function $f$ defined on a topological space $X$ is called $A$-continuous [15] if the preimage of every open subset of $\mathrm{R}$ belongs to $A$, where $A$ is a collection of subset of $X$. Most of the definitions of function used throughout this paper are consequences of the definition of $A$-continuity. However, for unknown concepts the reader may refer to $[2,5]$.

Hence, a real-valued function $f$ defined on a topological space $X$ is called perfectly continuous [14] (resp. contra-continuous [3]) if the preimage of every open subset of $\mathrm{R}$ is a clopen (i. e., open and closed) (resp. closed) subset of $X$.

We have a function is perfectly continuous if and only if it is continuous and contra-continuous.

Results of Kat ${ }^{2}$ etov $[6,7]$ concerning binary relations and the concept of an indefinite lower cut set for a real-valued function, which is due to Brooks [1], are used in order to give a necessary and sufficient conditions for the insertion of a perfectly continuous function between two comparable realvalued functions on the topological spaces that $\Lambda$-sets are open [10].

If $g$ and $f$ are real-valued functions defined on a space $X$, we write $g \leq f$ in case $g(x) \leq f(x)$ for all $x$ in $X$. The following definitions are modifications of conditions considered in [8].

A property $P$ defined relative to a real-valued function on a topological space is a $p c$-property provided that any constant function has property $P$ and provided that the sum of a function with property $P$ and any perfectly continuous function also has property $P$. If $P 1$ and $P 2$ are $p c-$ property, the following terminology is used: A space $X$ has the weak pc-insertion property for $(P 1, P 2)$ if and only if for any functions $g$ and $f$ on $X$ such that $g \leq f, g$ has property $P 1$ and $f$ has property $P 2$, then there exists a perfectly continuous function $h$ such that $g \leq h \leq f$.

In this paper, is given a sufficient condition for the weak $p c$-insertion property. Also, several insertion theorems are obtained as corollaries of these results. In addition, the insertion and strong insertion of a contracontinuous function between two comparable contra-precontinuous (contrasemi-continuous) functions have also recently considered by the author in $[11,12]$.

\section{The Main Result}

Before giving a sufficient condition for insertability of a perfectly continuous function, the necessary definitions and terminology are stated.

Let $(X, \tau)$ be a topological space, the family of all open, closed and clopen will be denoted by $O(X, \tau)$, $C(X, \tau)$ and $C l o(X, \tau)$, respectively.

Definition 2.1. Let $A$ be a subset of a topological space $(X, \tau)$. We define the subsets $A^{\Lambda}$ and $A^{V}$ as follows: 
$A^{\Lambda}=\bigcap\{O: O \supseteq A, O \in O(X, \tau)\}$ and $A^{V}=\cup\{F: F \subseteq A, F \in C(X, \tau)\}$.

In $[4,9,13], A^{\Lambda}$ is called the kernel of $A$.

Definition 2.2. Let $A$ be a subset of a topological space $(X, \tau)$. Respectively, we define the closure, interior, clo-closure and clo-interior of a set $A$, denoted by $C l(A), \operatorname{Int}(A), \operatorname{clo}(C l(A))$ and $\operatorname{clo}(\operatorname{Int}(A))$ as follows:

$C l(A)=\cap\{F: F \supseteq A, F \in C(X, \tau)\}, \operatorname{Int}(A)=\cup\{O: O \subseteq A, O \in O(X, \tau)\}, \operatorname{clo}(C l(A))=\bigcap\{F: F \supseteq A, F \in$ $C l o(X, \tau)\}$ and $\operatorname{clo}(\operatorname{Int}(A))=\cup\{O: O \subseteq A, O \in C l o(X, \tau)\}$.

If $(X, \tau)$ be a topological space whose $\Lambda^{-}$-sets are open, then respectively, we have $A^{V}, \operatorname{clo}(\operatorname{Cl}(A))$ are closed, clopen and $A^{\Lambda}, \operatorname{clo}(\operatorname{Int}(A))$ are open, clopen.

The following first two definitions are modifications of conditions considered in [6, 7].

Definition 2.3. If $\rho$ is a binary relation in a set $S$ then $\rho^{-}$is defined as follows: $x \rho^{-} y$ if and only if $y \rho$ $v$ implies $x \rho v$ and $u \rho x$ implies $u \rho y$ for any $u$ and $v$ in $S$.

Definition 2.4. A binary relation $\rho$ in the power set $P(X)$ of a topological space $X$ is called a strong binary relation in $P(X)$ in case $\rho$ satisfies each of the following conditions:

- If $A_{i} \rho B_{j}$ for any $i \in\{1, \ldots, m\}$ and for any $j \in\{1, \ldots, n\}$, then there exists a set $C$ in $P(X)$ such that $A_{i} \rho$ $C$ and $C \rho B_{j}$ for any $i \in\{1, \ldots, m\}$ and any $j \in\{1, \ldots, n\}$.

- If $A \subseteq B$, then $A \rho^{-} B$.

- If $A \rho B$, then $\operatorname{clo}(C l(A)) \subseteq B$ and $A \subseteq \operatorname{clo}(\operatorname{Int}(B))$.

The concept of a lower indefinite cut set for a real-valued function was defined by Brooks [1] as follows:

Definition 2.5. If $f$ is a real-valued function defined on a space $X$ and if $\{x \in X: f(x)<\ell\} \subseteq A(f, \ell) \subseteq\{x$ $\in X: f(x) \leq \ell\}$ for a real number $\ell$, then $A(f, \ell)$ is called a lower indefinite cut set in the domain of $f$ at the level $\ell$.

We now give the following main result:

Theorem 2.1. Let $g$ and $f$ be real-valued functions on a topological space $X$, in which $\Lambda$-sets are open, with $g \leq f$. If there exists a strong binary relation $\rho$ on the power set of $X$ and if there exist lower indefinite cut sets $A(f, t)$ and $A(g, t)$ in the domain of $f$ and $g$ at the level $t$ for each rational number $t$ such that if $t 1$ $<t 2$ then $A(f, t 1) \rho A(g, t 2)$, then there exists a perfectly continuous function $h$ defined on $X$ such that $g$ $\leq h \leq f$. Proof. Let $g$ and $f$ be real-valued functions defined on $X$ such that $g \leq f$. By hypothesis there exists a strong binary relation $\rho$ on the power set of $X$ and there exist lower indefinite cut sets $A(f, t)$ and $A(g, t)$ in the domain of $f$ and $g$ at the level $t$ for each rational number $t$ such that if $t 1<t 2$ then $A(f, t 1) \rho$ $A(g, t 2)$.

Define functions $F$ and $G$ mapping the rational numbers Q into the power set of $X$ by $F(t)=A(f, t)$ and $G(t)=A(g, t)$. If $t 1$ and $t 2$ are any elements of Q with $t 1<t 2$, then $F(t 1) \rho^{-} F(t 2), G(t 1) \rho^{-} G(t 2)$, and $F(t 1) \rho G(t 2)$. By Lemmas 1 and 2 of [7] it follows that there exists a function $H$ mapping Q into the power set of $X$ such that if $t 1$ and $t 2$ are any rational numbers with $t 1<t 2$, then $F(t 1) \rho H(t 2), H(t 1) \rho$ $H(t 2)$ and $H(t 1) \rho G(t 2)$.

For any $x$ in $X$, let $h(x)=\inf \{t \in \mathrm{Q}: x \in H(t)\}$.

We first verify that $g \leq h \leq f$ : If $x$ is in $H(t)$ then $x$ is in $G\left(t^{\prime}\right)$ for any $t^{\prime}>t$; since $x$ is in $G\left(t^{\prime}\right)=A\left(g, t^{\prime}\right)$ implies that $g(x) \leq t^{\prime}$, it follows that $g(x) \leq t$. Hence $g \leq h$. If $x$ is not in $H(t)$, then $x$ is not in $F\left(t^{\prime}\right)$ for any $t^{\prime}<t$; since $x$ is not in $F\left(t^{\prime}\right)=A\left(f, t^{\prime}\right)$ implies that $f(x)>t^{\prime}$, it follows that $f(x) \geq t$. Hence $h \leq f$.

Also, for any rational numbers $t 1$ and $t 2$ with $t 1<t 2$, we have $h^{-1}(t 1, t 2)=\operatorname{clo}(\operatorname{Int}(H(t 2))) \backslash$ $\operatorname{clo}(C l(H(t 1)))$. Hence $h^{-1}(t 1, t 2)$ is a clopen subset of $X$, i. e., $h$ is a perfectly continuous function on $X$.

The above proof used the technique of proof of Theorem 1 of [6].

\section{Applications}

The abbreviations $c, p c$ and $c c$ are used for continuous, perfectly continuous and contra-continuous, respectively. 
Before stating the consequences of theorems 2.1, we suppose that $X$ is a topological space that $\Lambda^{-}$-sets are open.

Corollary 3.1. If for each pair of disjoint closed (resp. open) sets $F 1, F 2$ of $X$, there exist clopen sets $G 1$ and $G 2$ of $X$ such that $F 1 \subseteq G 1, F 2 \subseteq G 2$ and $G 1 \cap G 2=\varnothing$ then $X$ has the weak $p c$-insertion property for $(c, c)$ ( resp.

$(c c, c c))$.

Proof. Let $g$ and $f$ be real-valued functions defined on the $X$, such that $f$ and $g$ are $c$ (resp. $c c$ ), and $g \leq$ $f$.If a binary relation $\rho$ is defined by $A \rho B$ in case $C l(A) \subseteq \operatorname{Int}(B)\left(\right.$ resp. $\left.A^{\Lambda} \subseteq B^{V}\right)$, then by hypothesis $\rho$ is a strong binary relation in the power set of $X$. If $t 1$ and $t 2$ are any elements of Q with $t 1<t 2$, then

$A(f, t 1) \subseteq\{x \in X: f(x) \leq t 1\} \subseteq\{x \in X: g(x)<t 2\} \subseteq A(g, t 2) ;$

since $\{x \in X: f(x) \leq t 1\}$ is a closed (resp. open) set and since $\{x \in X: g(x)<t 2\}$ is an open (resp. closed) set, it follows that $C l(A(f, t 1)) \subseteq \operatorname{Int}(A(g, t 2))\left(\right.$ resp. $\left.A(f, t 1)^{\Lambda} \subseteq A(g, t 2)^{V}\right)$. Hence $t 1<t 2$ implies that $A(f, t 1)$ $\rho A(g, t 2)$. The proof follows from Theorem 2.1.

Corollary 3.2. If for each pair of disjoint closed (resp. open) sets $F 1, F 2$, there exist clopen sets $G 1$ and $G 2$ such that $F 1 \subseteq G 1, F 2 \subseteq G 2$ and $G 1 \cap G 2=\emptyset$ then every continuous (resp. contra-continuous) function is perfectly continuous.

Proof. Let $f$ be a real-valued continuous (resp. contra-continuous) function defined on the $X$. By setting $g=f$, then by Corollary 3.1, there exists a perfectly continuous function $h$ such that $g=h=f$.

Corollary 3.3. If for each pair of disjoint closed (resp. open) sets $F 1, F 2$ of $X$, there exist clopen sets $G 1$ and $G 2$ of $X$ such that $F 1 \subseteq G 1, F 2 \subseteq G 2$ and $G 1 \cap G 2=\emptyset$ then $X$ has the $p c$-insertion property for $(c, c)$ (resp. $(c c, c c)$ ). Proof. Let $g$ and $f$ be real-valued functions defined on the $X$, such that $f$ and $g$ are $c$ (resp. $c c$ ), and $g<f$. Set $h=(f+g) / 2$, thus $g<h<f$, and by Corollary 3.2, since $g$ and $f$ are perfectly continuous functions hence $h$ is a perfectly continuous function.

Corollary 3.4. If for each pair of disjoint subsets $F 1, F 2$ of $X$, such that $F 1$ is closed and $F 2$ is open, there exist clopen subsets $G 1$ and $G 2$ of $X$ such that $F 1 \subseteq G 1, F 2 \subseteq G 2$ and $G 1 \cap G 2=\emptyset$ then $X$ have the weak $p c$-insertion property for $(c, c c)$ and $(c c, c)$.

Proof. Let $g$ and $f$ be real-valued functions defined on the $X$, such that $g$ is $c$ (resp. $c c$ ) and $f$ is $c c$ (resp. c), with $g \leq f$.If a binary relation $\rho$ is defined by $A \rho B$ in case $A^{\Lambda} \subseteq \operatorname{Int}(B)$ (resp. $C l(A) \subseteq B^{V}$ ), then by hypothesis $\rho$ is a strong binary relation in the power set of $X$. If $t 1$ and $t 2$ are any elements of Q with $t 1$ $<t 2$, then

$A(f, t 1) \subseteq\{x \in X: f(x) \leq t 1\} \subseteq\{x \in X: g(x)<t 2\} \subseteq A(g, t 2) ;$

since $\{x \in X: f(x) \leq t 1\}$ is an open (resp. closed) set and since $\{x \in X: g(x)<t 2\}$ is an open (resp. closed) set, it follows that $A(f, t 1)^{\Lambda} \subseteq \operatorname{Int}(A(g, t 2))$ (resp. $\left.C l(A(f, t 1)) \subseteq A(g, t 2)^{V}\right)$. Hence $t 1<t 2$ implies that

$A(f, t 1) \rho A(g, t 2)$. The proof follows from Theorem 2.1.

\section{ACKNOWLEDGEMENT}

This research was partially supported by Centre of Excellence for Mathematics (University of Isfahan).

\section{REFERENCES}

[1] F. Brooks, Indefinite cut sets for real functions, Amer. Math. Monthly, 78 (1971), 1007-1010.

[2] J. Dontchev, The characterization of some peculiar topological space via $\alpha-$ and $\beta$-sets, Acta Math. Hungar., 69 (1-2)(1995), 67-71.

[3] J. Dontchev, Contra-continuous functions and strongly S-closed space, Intrnat. J. Math. Math. Sci., 19 (2)(1996), 303-310.

[4] J. Dontchev, and H. Maki, On sg-closed sets and semi- $\lambda$-closed sets, Questions Answers Gen. Topology, 15 (2)(1997), 259-266.

[5] M. Ganster and I. Reilly, A decomposition of continuity, Acta Math. Hungar., 56 (3-4)(1990), 299-301.

[6] M. Kat ${ }^{`}$ etov, On real-valued functions in topological spaces, Fund. Math., 38 (1951), 85-91. 
[7] M. Katetov, Correction to, "On real-valued functions in topological spaces”, Fund. Math., 40 (1953), $203-$ 205.

[8] E. Lane, Insertion of a continuous function, Pacific J. Math., 66 (1976) , 181-190.

[9] S. N. Maheshwari and R. Prasad, On $R_{O S}-$ spaces, Portugal. Math., 34 (1975), 213-217.

[10] H. Maki, Generalized $\Lambda$-sets and the associated closure operator, The special Issue in commemoration of Prof. Kazuada IKEDA's Retirement, (1986), 139-146.

[11] M. Mirmiran, Insertion of a contra-continuous function between two comparable contra-precontinuous (contra-semi-continuous) functions, International Journal of Scientific and Innovative Mathematical Research, 7(10)(2019), 34-40.

[12] M. Mirmiran, Strong insertion of a contra-continuous function between two comparable contraprecontinuous (contra-semi-continuous) functions, International Journal of Scientific and Innovative Mathematical Research, 7(11)(2019), 12-16.

[13] M. Mrsevic, On pairwise $R$ and pairwise $R 1$ bitopological spaces, Bull. Math. Soc. Sci. Math. R. S. Roumanie, 30 (1986), 141-145.

[14] T. Noiri, Super-continuity and some strong forms of continuity, Indian J. Pure Appl. Math., 15 (1984), 241250.

[15] M. Przemski, A decomposition of continuity and $\alpha$-continuity, Acta Math. Hungar., 61 (1-2)(1993), 93-98.

Citation: MAJID MIRMIRAN, (2019). Weak Insertion of a Perfectly Continuous Function. International Journal of Scientific and Innovative Mathematical Research (IJSIMR), 7(12), pp. 6-9. http://dx.doi.org/ 10.20431/2347 -3142.0712002

Copyright: (c) 2019 Authors, this is an open-access article distributed under the terms of the Creative Commons Attribution License, which permits unrestricted use, distribution, and reproduction in any medium, provided the original author and source are credited. 